\title{
SNR Analysis of RoF Systems based on 64-QAM Including the Impact of Dispersion and Phase Noise
}

\author{
Shelly Singla \\ Guru Jambheshwar University of Science and \\ Terchnology, \\ Hisar
}

\begin{abstract}
In this paper, the performance of radio-over-fiber (RoF) links employing intensity modulation has been investigated in terms of signal to noise ratio (SNR). An analytical model including dispersion, laser and radio frequency (RF) oscillator phase noise is constructed to estimate the SNR performance for 64- quadrature amplitude modulation (QAM) based RoF systems. It has been observed that the RF oscillator line-width and laser line-width affect the SNR significantly. SNR decreases monotonously as the value of laser line width increases but for increased RF oscillator line widths specifically above $1 \mathrm{~Hz}$, the SNR degrades to a great extent [20 dB] which is not desirable for efficient communication system.
\end{abstract}

\section{Keywords}

RoF, SNR, QAM, Phase noise, Dispersion.

\section{INTRODUCTION}

Radio over fiber is becoming an increasingly attractive technology for implementing broadband in building wireless access coverage, such as in corporate office buildings, shopping malls and airports [1]. The direct modulation based scheme of generation is simple but suffers from a laserfrequency chirp effect leading to the performance degradation. External modulation scheme can be used to improve the system performance [2]. The conventional double sideband transmission technique is affected by fiber chromatic dispersion severely. Single side band (SSB) modulation can be used to overcome the deteriorating effects caused by chromatic dispersion. In a radio-over-fiber system which carries millimeter-wave (MM) signals, the chromatic dispersion limited transmission distance also gets extended by the use of SSB technique [3], whereas the radio-spectrumlimited capacity can be overcome by using multilevel modulation techniques such as $M$-ary quadrature amplitude modulation (M-QAM) techniques [4]-[6]. Regardless of the number of constellation points, all quadrature-amplitude modulation (QAM) signals can be generated using a single dual-drive Mach-Zehnder modulator [7]. The QAM signal generation is greatly simplified with the usage of only one dual-drive modulator. Multilevel modulation signals like QAM improve the spectral efficiency of a optical communication system. Here, an analytical model for the signal to noise ratio (SNR) for 64-QAM based RoF system has been developed including the impact of dispersion, phase noise from laser and RF oscillator.

\author{
Sandeep K. Arya \\ Guru Jambheshwar University of Science and \\ Terchnology, \\ Hisar
}

\section{THEORETICAL MODELING AND SNR ANALYSIS}

The QAM signal consists of two phase quadrature carriers each of which is modulated by a set of discrete amplitudes. An M-array QAM signal can be generated using dual electrode Mach-Zehnder modulators (DE-MZM). The Marray QAM signal generated using dual electrode MZM is given as [7]:

$E_{Q A M}=\frac{E_{\text {in }}}{2}\left[e^{j \varphi_{1}}-e^{j \varphi_{2}}\right]$

Where $\varphi_{1}=\pi \frac{V_{1}}{V_{\pi}}, \varphi_{2}=\pi \frac{V_{2}}{V_{\pi}}+\pi$,

Here, $V_{1}$ and $V_{2}$ are the signals from RF oscillator. The phase of $V_{1}$ and $V_{2}$ are carefully chosen to generate quadrature signal in the output. The RF signal is optically modulated by the laser source with an MZM. The optical signals from the laser and the RF oscillator are modeled as [8]:

$$
\mathrm{X}_{\mathrm{LD}}(\mathrm{t})=A \cdot \mathrm{e}^{\mathrm{j}\left[\mathrm{w}_{\mathrm{d}} \mathrm{t}+\emptyset_{\mathrm{d}}(\mathrm{t})\right]}
$$

and

$$
x_{0}(t)=V_{0} \cdot \cos \left[w_{0} t+\emptyset_{0}(t)\right]
$$

where $A_{d}, V_{\mathrm{o}}$ are amplitudes from the laser source and the $\mathrm{RF}$ oscillator signal, $\omega_{\mathrm{d}}$ is angular frequency of the laser signal, $w_{0}$ is the RF oscillator signal, $\emptyset_{d}(t), \emptyset_{0}(t)$ are phase-noise processes. The electrical output of QAM signal generated using dual electrode MZM is modeled as [9]:

$$
\begin{aligned}
& \mathrm{E}_{\mathrm{QAM}}(0, \mathrm{t})= \\
& A_{d} \mathrm{~L}_{\mathrm{MZM}}\left[\mathrm{J}_{0}(\beta \pi) \mathrm{e}^{\mathrm{j}\left[\mathrm{w}_{\mathrm{d}} \mathrm{t}+\emptyset_{\mathrm{d}}(\mathrm{t})+\frac{\pi}{4}\right]}-\right. \\
& \left.\sqrt{2} \mathrm{~J}_{1}(\beta \pi) \mathrm{e}^{\mathrm{j}\left[\mathrm{w}_{\mathrm{d}} \mathrm{t}+\emptyset_{\mathrm{d}}(\mathrm{t})+\mathrm{w}_{0} \mathrm{t}+\emptyset_{0}(\mathrm{t})\right]}\right]
\end{aligned}
$$

$$
\text { Where } \beta \text { is } \frac{V_{0}}{\sqrt{2} V_{\pi}} \text {, and }
$$

$V_{\pi}$ is switching voltage of MZM, $\mathrm{L}_{\mathrm{MZM}}$ is insertion loss 
After the transmission of signal over a length of $\mathrm{L} \mathrm{km}$, the signal can be represented as:

$$
\begin{aligned}
& \mathrm{E}_{\mathrm{QAM}}(\mathrm{L}, \mathrm{t}) \\
& =A_{d} \mathrm{~L}_{\mathrm{MZM}} \mathrm{L}_{\mathrm{add}} 10^{\frac{\alpha \mathrm{L}}{20}} \mathrm{~J}_{0}(\beta \pi)\left[\mathrm{e}^{\mathrm{j}\left(\mathrm{w}_{\mathrm{d}} \mathrm{t}+\emptyset_{\mathrm{d}}\left(\mathrm{t}-\tau_{0}\right)-\emptyset_{1}+\frac{\pi}{4}\right)}\right. \\
& \left.-\frac{\sqrt{2 \mathrm{~J}_{1}(\beta \pi)}}{\mathrm{J}_{0}(\beta \pi)} \mathrm{e}^{\mathrm{j}\left(\mathrm{w}_{\mathrm{d}} \mathrm{t}+\emptyset_{\mathrm{d}}\left(\mathrm{t}-\tau_{+}\right)+\mathrm{w}_{0} \mathrm{t}+\emptyset_{0}\left(\mathrm{t}-\tau_{+}\right)-\emptyset_{2}\right)}\right]
\end{aligned}
$$

where $L_{\text {add }}$ is additional loss in the optical link, $\alpha$ is standard single mode fiber loss, $L$ is transmission distance, $\tau_{0}$ and $\tau_{+}$ are group delays for center angular frequency $\left(\mathrm{W}_{\mathrm{d}}\right)$ and upper sideband frequency $\left(\mathrm{w}_{\mathrm{d}}+\mathrm{w}_{0}\right), \emptyset_{1}$ and $\emptyset_{2}$ are phase-shift parameters for specific frequencies due to the chromatic dispersion.

The power signal received at the receiver based on power spectral density function is given as :

$P_{r c d} \cong \frac{4 r^{2} \alpha_{1}^{2} A_{D}^{4}}{\pi} e^{-2 \gamma_{t} \tau} \cdot \tan ^{-1}\left(\frac{\pi B_{0}}{2 \gamma_{0}}\right)$

Where $r$ is the resposivity, $\gamma_{t}$ is total linewidth, $2 \gamma_{t}=2 \gamma_{d}+2 \gamma_{o}, \gamma_{d}$ is $\pi \Delta_{d}, \gamma_{o} / 2$ is $\pi \Delta_{o}$, $\Delta_{d}$ is laser line-width, $\Delta_{o}$ is RF oscillator line-width, $\alpha_{1}$ is the loss due to normalized RF voltage, $A_{D}$ is a constant dependent on laser source amplitude and losses, $B_{0}$ is electrical filter bandwidth and $\tau$ is the differential delay due to the chromatic dispersion which is given as:

$$
\tau=D L \lambda^{2}\left(f_{o} / c\right)
$$

Where $\mathrm{D}$ is dispersion parameter, $\mathrm{L}$ is the transmission distance, $\lambda$ is the operation wavelength and $f_{o}$ is the carrier frequency.

Signal to noise ratio $(S N R)$ of the system can be given as:

$$
\begin{aligned}
S N R= & \frac{\text { Signal Power }}{\text { Noise Power }} \\
& S N R=\frac{2 r^{2} A_{D}{ }^{4} \alpha_{1}^{2} p}{N_{a} \frac{\gamma R F}{\pi} \tan \left[\frac{\pi}{2} \cdot p \cdot \exp \left(2 \gamma_{t} \tau\right)\right]}
\end{aligned}
$$

Where $\mathrm{p}$ is the percentage of power received, $N_{a}$ is the additive white gaussian noise power spectral density.

\section{RESULTS AND DISCUSSION}

The impact of phase noise due to laser line-width and RF oscillator line-width on SNR has been studied in the presence of dispersion. Simulations have been carried out for 64-QAM signal generated through DE-MZM. Table 1 summarises various simulation parameters selected to study the impact of dispersion and phase noise on SNR.

Table 1: Simulation Parameters for obtaining SNR

\begin{tabular}{|c|c|}
\hline Responsivity $(r)$ & $0.7 \mathrm{~A} / \mathrm{W}$ \\
\hline Switching Voltage $\left(V_{\pi}\right)$ & $2.5 \mathrm{~V}$ \\
\hline AWGN Noise & $10^{-11}$ \\
\hline Dispersion Parameter (D) & $17 \mathrm{ps} / \mathrm{km} . \mathrm{nm}$ \\
\hline Fiber Loss $(\alpha)$ & $0.2 \mathrm{~dB} / \mathrm{km}$ \\
\hline
\end{tabular}

Fig. 1 shows the variation of the SNR with laser line-width for 64-QAM signal. The SNR is plotted against laser line-width varying from 0 to $600 \mathrm{MHz}$ for four different values of RF oscillator line-width i.e. $0.5 \mathrm{~Hz}, 0.8 \mathrm{~Hz}, 1 \mathrm{~Hz}$ and $1.2 \mathrm{~Hz}$. The graph has been drawn for a $10 \mathrm{~km}$ transmission distance including the impact of dispersion of $17 \mathrm{ps} / \mathrm{km} . \mathrm{nm}$. It can be seen that SNR decreases monotonously with increase in the laser line-width at defferent values of RF line-width. SNR decreases from $15.1 \mathrm{~dB}$ to $13.9 \mathrm{~dB}$ when laser line-width is varied from 0 to $600 \mathrm{MHz}$ for $0.5 \mathrm{~Hz} \mathrm{RF}$ line-width. It can be seen from the graph that laser possessing line-width upto 10 $\mathrm{MHz}$ and $\mathrm{RF}$ oscillator upto $1 \mathrm{~Hz}$ are desirable for a $10 \mathrm{~km}$ transmission distance.

The effect of the RF line-width on the SNR of the system is shown in Fig. 2. The results obtained show an exponential decrement in SNR with the increase in RF line-width. Plots have been drawn for different values of laser line-width i.e. $100 \mathrm{MHz}, 300 \mathrm{MHz}$ and $600 \mathrm{MHz}$ including the impact of dispersion $17 \mathrm{ps} / \mathrm{km} . \mathrm{nm}$. It can be found from the results that good quality communication is achievable upto RF line-width value of $1 \mathrm{~Hz}$. After this SNR performance degrades very badly. For the $5 \mathrm{~Hz} \mathrm{RF}$ line-width, the SNR decreases further.

\section{CONCLUSION}

The effect of the phase noise induced from the laser linewidth and the RF oscillator line-width on the SNR of the 64Ary QAM RoF systems has been studied. In most of the light wave communication systems, the lower threshold for the SNR is less than $10 \mathrm{~dB}$. If the SNR decreases below this value, the link does not support high quality multimedia services. Greater the phase noise, the more is noise penetration into the system and hence poorer is the SNR. The effect of the RF oscillator line-width on the system SNR is also studied. To achieve better SNR performance, the RF oscillator line-width should be below $1 \mathrm{~Hz}$. This means we need to use highly stable RF oscillators. It can also be depicted that for RoF links, the effect of the RF oscillator linewidth on the system SNR is more pronounced than that of the laser line width. 


\section{REFERENCES}

[1] M. Sauer, A. Kobyakov, and J. George, "Radio-overfiber for picocellular network architectures ," Journal of lightwave technology., vol. 25, no. 11, pp.3301-3320, Nov. 2007.

[2] A. J. Seeds and K. J. Williams, "Microwave photonics," Journal of lightwave technology., vol. 24, no. 12, pp. 4628-4641, Dec. 2006.

[3] G. H. Smith, D. Novak, and Z. Ahmed, "Overcoming chromatic-dispersion effects in fiber-wireless systems incorporating external modulators," IEEE Trans. Microw. Theory Tech., vol. 45, no. 8, pt. 2, pp. 14101415, Aug. 1997.

[4] V. J. Urick, J. X. Qiu, and F. Bucholtz, "Wide-band QAM-over-fiber using phase modulation and interferometric demodulation," IEEE Photon. Technol. Lett., vol. 16, no. 10, pp. 2374-2376, Oct. 2004.

[5] M. A. Piqueras, B. Vidal, J. L. Corral, V. Polo, A. Martinez, and J.Marti, "Direct photonic generation of electrical vector modulations at microwave/millimeterwave frequencies," IEEE Photon. Technol. Lett., vol. 17, no. 9, pp. 1947-1949, Sep. 2005.

[6] J. Hongo, K. Kasai, M. Yoshida, and M. Nakazawa, "1Gsymbol/s 64-QAM coherent optical transmission over 150 km," IEEE Photon. Technol. Lett., vol. 19, no. 9, pp. 638-640, May 2007.

[7] Keang-Po Ho and Han-Wei Cuei, "Generation of Arbitrary Quadrature Signals Using One Dual-Drive Modulator", Journal of lightwave technology, Vol. 23, No. 2, February 2005.

[8] G. P. Aggarwal, Fiber-Optic Communication Systems, New York, Wiley, 2005.

[9] Vishal Sharma, Amarpal Singh, Ajay K. Sharma, "Simulative investigation on the impact of laser-spectral width in single-tone radio-over-fiber transmission system using optical single side-band technique", Optics and Lasers in Engineering, Vol.47, pp-1145-1149, July 2009.

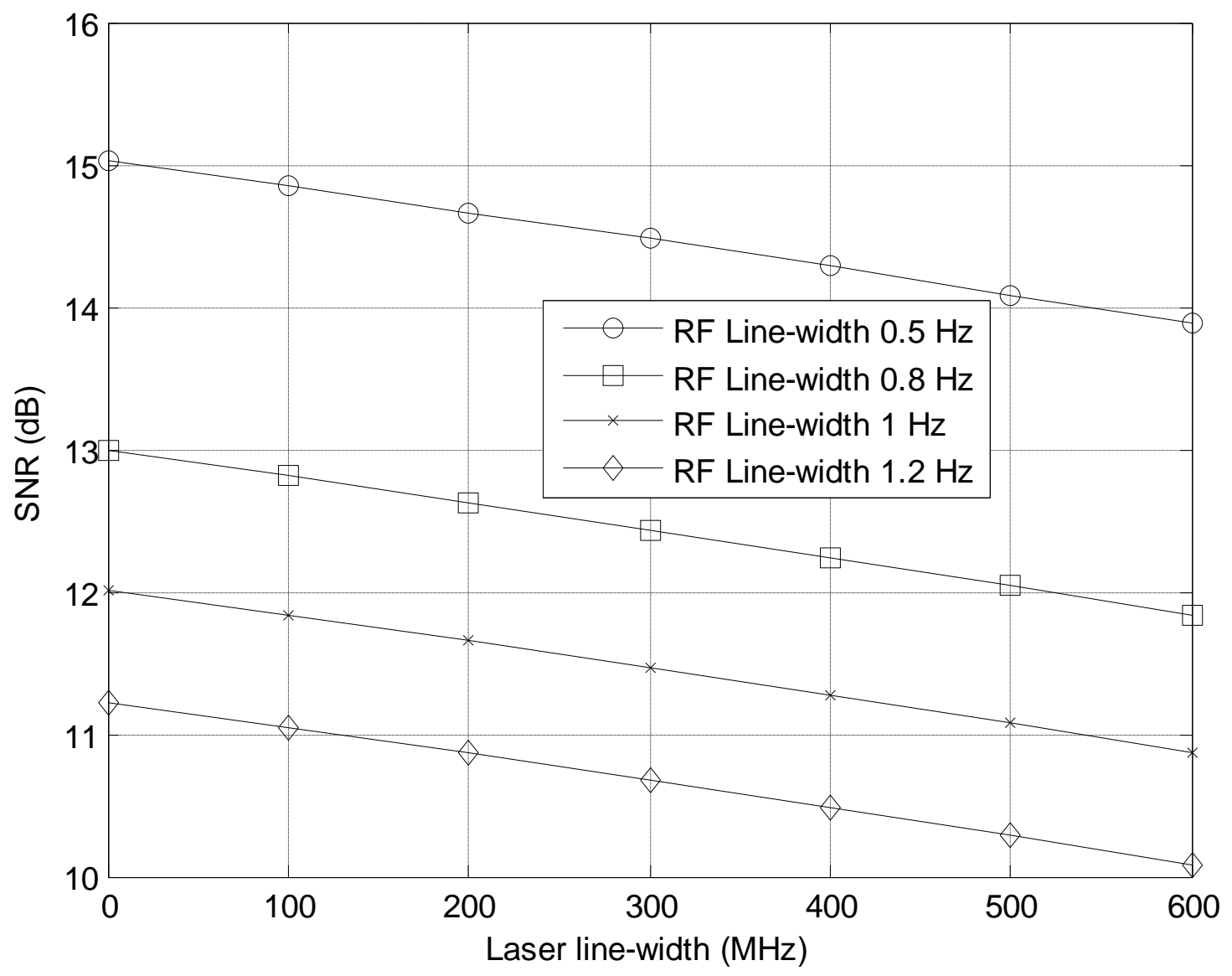

Fig 1: SNR as a function of laser line-width for varying RF line-width 


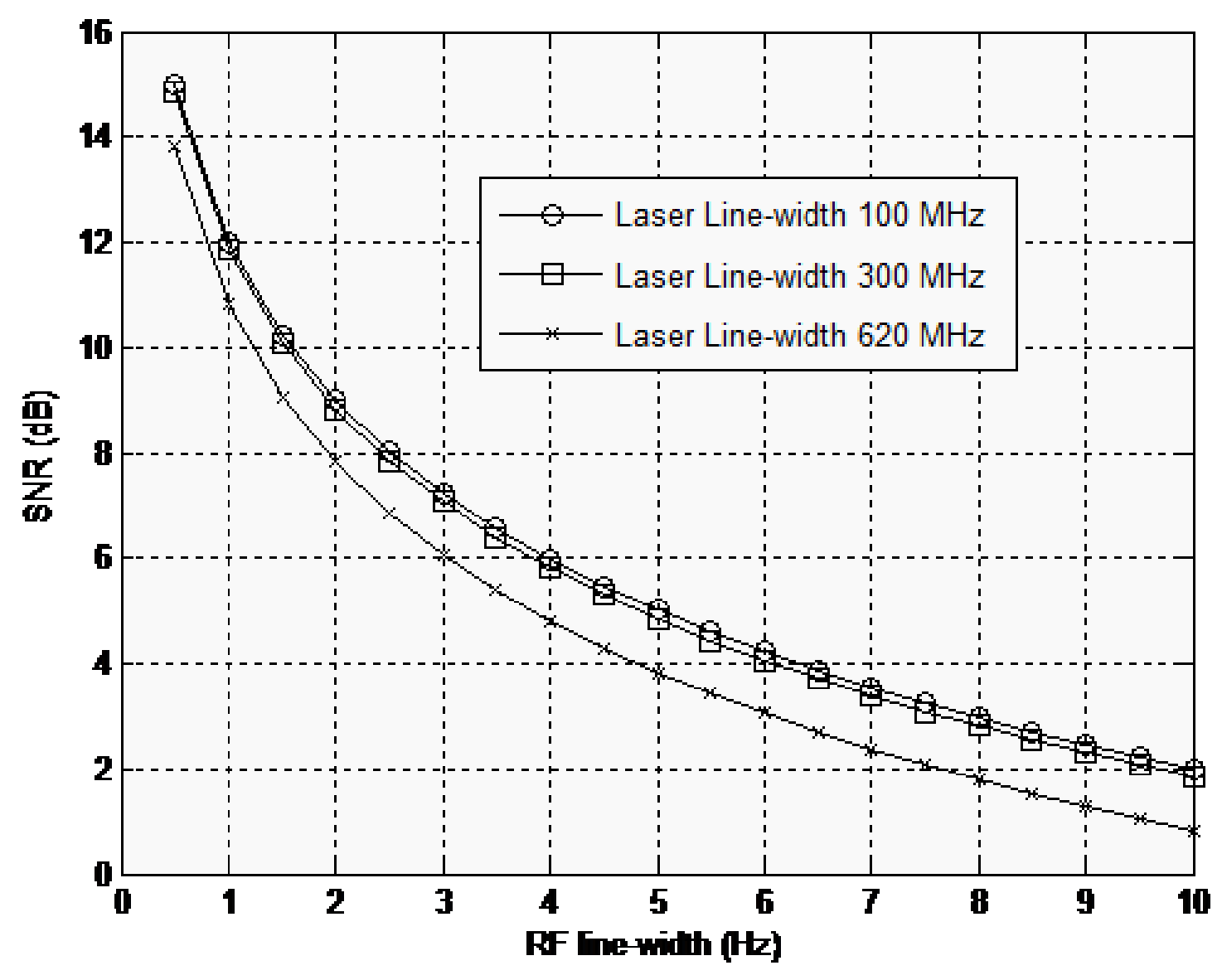

Fig 2: SNR as a function of RF Line-width varying laser line-width 